\title{
Hepatic Steatosis in Lean Patients: Risk Factors and Impact on Mortality
}

\author{
Lukas W. Unger ${ }^{1,2} \cdot$ Bernadette Forstner $^{1} \cdot$ Moritz Muckenhuber $^{1} \cdot$ Katharina Scheuba $^{1} \cdot$ Ernst Eigenbauer $^{3}$. \\ Bernhard Scheiner ${ }^{2,4} \cdot$ Nikolaus Pfisterer $^{2,5} \cdot$ Rafael Paternostro $^{2,4} \cdot$ Michael Trauner $^{4} \cdot$ Mattias Mandorfer $^{2,4}$. \\ Thomas Reiberger ${ }^{2,4}$
}

Received: 27 August 2019 / Accepted: 5 December 2019 / Published online: 24 December 2019

(c) The Author(s) 2019

\begin{abstract}
Background The prognostic impact of liver steatosis in obese patients is well established. Limited data on the risk factors for and impact of hepatic steatosis in lean patients are available.

Aims Assess risk factors for liver steatosis in lean patients and investigate its impact on survival.

Methods Patients without viral hepatitis and with a BMI $\leq 25 \mathrm{~kg} / \mathrm{m}^{2}$ undergoing liver stiffness measurement (LSM) and controlled attenuation parameter (CAP) by transient elastography were retrospectively identified. Clinical characteristics and laboratory test results were obtained at the time of LSM/CAP measurement. National death registry data were obtained in order to assess survival.

Results Among $n=218$ lean patients, $n=97$ (34.5\%) showed significant liver steatosis (CAP $\geq 268 \mathrm{~dB} / \mathrm{m}$ ), while $n=184$ $(65.5 \%)$ had no or just mild steatosis $(\mathrm{CAP}<268 \mathrm{~dB} / \mathrm{m})$. Patients with steatosis had higher GGT $(238.0( \pm 450.3)$ vs. $112.1( \pm 180.0) \mathrm{IU} / \mathrm{mL} ; p=0.013)$, AST $(63( \pm 67.4)$ vs. 38.5( \pm 32.9$) \mathrm{IU} / \mathrm{mL} ; p=0.001)$, ALT $(59.1( \pm 58.8)$ vs. $44.3( \pm 52.7)$ $\mathrm{IU} / \mathrm{mL} ; p=0.048)$ and triglyceride levels $(120.1( \pm 80.3) \mathrm{vs.} 96.1( \pm 58.2) \mathrm{mg} / \mathrm{dL} ; p=0.014)$, and showed a trend toward more severe fibrosis (LSM 15.6 $( \pm 19.5)$ vs. $12.0( \pm 15.7) \mathrm{kPa} ; p=0.115)$. In multivariate binary logistic regression analysis, only serum uric acid levels were independently associated with liver steatosis (odds ratio 1.43 per unit $\mathrm{mg} / \mathrm{dL} ; 95 \%$ CI 1.001-2.054; $p=0.049)$. During a mean follow-up of 38.9( \pm 10.6$)$ months, $n=14$ patients $(5.0 \%)$ died. In the absence of advanced fibrosis, survival after 1 year was similar in patients without (98.7\%) and with (98.6\%) significant steatosis. Patients with advanced fibrosis had worse 1-year survival without concomitant significant steatosis (84.8\%) than patients with steatosis $(95.8 \%$; log-rank $p<0.001)$.
\end{abstract}

Conclusions High serum uric acid levels increase the risk of liver steatosis in lean patients. Liver fibrosis but not hepatic steatosis is a risk factor for impaired survival in lean patients.

Keywords Hyperuricemia $\cdot$ Dyslipidemia $\cdot$ Chronic liver disease $\cdot$ Outcome

Abbreviations

A1AD alpha-1 antitrypsin deficiency

AIH autoimmune hepatitis

Electronic supplementary material The online version of this

ALD alcoholic liver disease

article (https://doi.org/10.1007/s10620-019-06000-y) contains

supplementary material, which is available to authorized users.

ALT alanine aminotransferase

Thomas Reiberger

thomas.reiberger@meduniwien.ac.at

1 Division of General Surgery, Department of Surgery, Medical University of Vienna, Währinger Gürtel 18-20, Vienna, Austria

2 Vienna Hepatic Hemodynamic Laboratory, Medical University of Vienna, Währinger Gürtel 18-20, Vienna, Austria
3 IT-Systems and Communications, Medical University of Vienna, Währinger Gürtel 18-20, Vienna, Austria

4 Division of Gastroenterology and Hepatology, Department of Internal Medicine III, Medical University of Vienna, Währinger Gürtel 18-20, 1090 Vienna, Austria

5 Krankenanstalt Rudolfstiftung, Krankenanstaltenverbund Wien (KAV), Vienna, Austria 


$\begin{array}{ll}\text { AST } & \text { aspartate aminotransferase } \\ \text { BMI } & \text { body mass index } \\ \text { CAP } & \text { controlled attenuation parameter } \\ \text { CF } & \text { cystic fibrosis } \\ \text { GGT } & \text { gamma-glutamyltransferase } \\ \text { HBV } & \text { hepatitis B virus } \\ \text { HCV } & \text { hepatitis C virus } \\ \text { HFE } & \text { hemochromatosis } \\ \text { LSM } & \text { liver stiffness measurement } \\ \text { NAFLD } & \text { Nonalcoholic fatty liver disease } \\ \text { NASH } & \text { Nonalcoholic steatohepatitis } \\ \text { NFS } & \text { NAFLD fibrosis score } \\ \text { PBC } & \text { primary biliary cholangitis } \\ \text { PSC } & \text { primary sclerosing cholangitis }\end{array}$

\section{Introduction}

The global prevalence of obesity and nonalcoholic fatty liver disease (NAFLD) is continuously increasing [1,2] with both diseases representing a significant individual as well as socioeconomic burden [3]. While NAFLD is usually diagnosed in individuals with obesity, type 2 diabetes mellitus, dyslipidemia [4], and physical inactivity [5], limited evidence is available for the prevalence of steatosis and NAFLD in lean patients who do not show typical risk factors related to the metabolic syndrome [6].
Abdominal ultrasound is the first-line test for diagnosing liver steatosis; however, liver stiffness measurement (LSM) with controlled attenuation parameter (CAP) allows simultaneous screening for liver fibrosis and steatosis in specialized centers [7]. Importantly, simple steatosis in NAFLD patients may progress to nonalcoholic steatohepatitis (NASH) with subsequent fibrosis [8-10]. Thus, the identification of risk factors for hepatic steatosis in lean patients seems clinically relevant.

Since only limited data on the potentially different NAFLD risk profiles in lean patients are available [11-15], we aimed (i) to identify risk factors for hepatic steatosis and (ii) to assess its impact on survival in lean patients.

\section{Materials and Methods}

\section{Study Design and Patient Selection (Fig. 1)}

All patients who underwent liver stiffness measurement (LSM) with controlled attenuation parameters (CAP) using a Fibroscan ${ }^{\circledR}$ device (EchoSens, Paris, France) between January 2013 and October 2016 in the liver outpatient clinic of the Medical University of Vienna were evaluated for inclusion. After exclusion due to obesity, missing BMI, or chronic liver disease other than NAFLD, ALD, AIH or no liver disease, 281 patients were eligible for the final analysis. Of

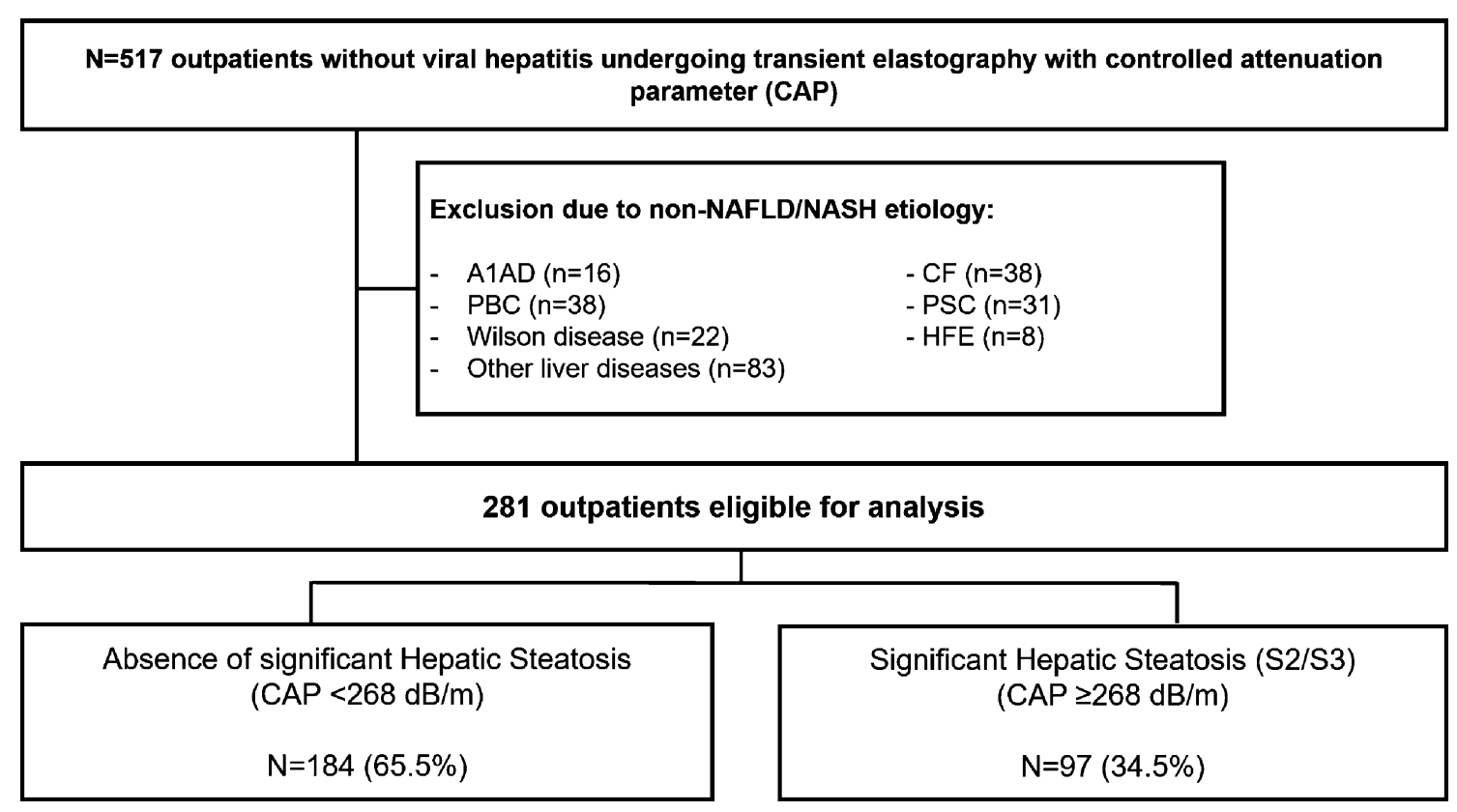

Fig. 1 Patient flowchart. For final analysis, 184 patients with $\mathrm{BMI} \leq 25 \mathrm{~kg} / \mathrm{m}^{2}$ and no steatosis as assessed by CAP and 97 patients with $\mathrm{BMI} \leq 25 \mathrm{~kg} / \mathrm{m}^{2}$ and significant steatosis as assessed by CAP were included. Abbreviations: A1AD, alpha-1 antitrypsin deficiency; $\mathrm{AIH}$, autoimmune hepatitis; ALD, alcoholic liver disease; CAP, con- trolled attenuation parameters; $\mathrm{CF}$, cystic fibrosis; $\mathrm{HBV}$, hepatitis B virus; $\mathrm{HCV}$, hepatitis $\mathrm{C}$ virus; HFE, hemochromatosis; NAFLD, nonalcoholic fatty liver disease; NASH, nonalcoholic steatohepatitis; PBC, primary biliary cholangitis; PSC, primary sclerosing cholangitis 
these, $184(65.5 \%)$ had no significant steatosis according to $\mathrm{CAP}(\mathrm{S} 0 / \mathrm{S} 1$; CAP $<268 \mathrm{~dB} / \mathrm{m})$ and 97 (34.5\%) had significant steatosis $(\mathrm{S} 2 / \mathrm{S} 3 ; \mathrm{CAP} \geq 268 \mathrm{~dB} / \mathrm{m})$.

\section{Assessment of Advanced Liver Fibrosis, Hepatic Steatosis, and Laboratory Results}

A Fibroscan ${ }^{\circledR}$ device (EchoSens, Paris, France) was used for noninvasive liver stiffness measurement (LSM) with concomitant evaluation of steatosis using CAP, as previously described [16]. In brief, overnight fasting and a total number of ten valid measurements were required. LSM evaluation of fibrosis and steatosis was performed due to clinical indications. A cutoff value of $\geq 15 \mathrm{kPa}$ defined the presence of advanced chronic liver disease (i.e., advanced fibrosis/ cirrhosis), as suggested by the Baveno VI consensus [17].

After extensive evaluation, the diagnosis of chronic liver disease was autoimmune hepatitis (AIH) in 50 patients (17.8\%), alcoholic liver disease in 52 patients $(18.5 \%)$, cryptogenic liver disease in 21 patients $(7.5 \%)$, NAFLD in 74 patients $(26.3 \%)$, and no diagnosed liver disease despite extensive evaluation in 84 patients (29.9\%). Laboratory results were obtained within 3 months of LSM.

\section{Assessment of Metabolic Risk Factors}

Diabetes mellitus, arterial hypertension, and dyslipidemia were assessed during routine clinical examination. None of the included patients suffered from diabetes mellitus or was on any antidiabetic medication. Forty-one patients (14.9\%) were taking antihypertensive medication (betablockers, alphablockers, angiotensin-converting enzyme inhibitors, calcium channel blockers or AT-1 receptor blockers), and 26 were on statin therapy due to dyslipidemia (9.3\%).

Patient's medical records were screened for the use of allopurinol and diuretics (i.e., loop diuretics, thiazides or potassium-sparing diuretics).

\section{Survival and Follow-Up}

Patients were included at the time of LSM. For survival analysis, the national death registry was consulted to obtain complete data on mortality in our cohort. If patients were alive according to the national death registry, they were censored at the time of last outpatient visit documented in their electronic medical record.

\section{Institutional Review Board and Informed Consent}

The retrospective cohort study conformed to the ethical guidelines of the 1975 Declaration of Helsinki (6th revision, 2008) as reflected in a priori approval by the Medical
University of Vienna's institutional review board (EK-Nr. 2013/2016; https://ekmeduniwien.at/core/catalog/2016/).

\section{Statistical Analysis}

Differences in proportions between groups were evaluated using Chi-squared or Fisher's exact tests whenever appropriate. For numerical variables and comparisons between two groups, Student's $t$ test or Mann-Whitney $U$ test was used, as applicable.

To investigate the impact of different factors on the presence of significant steatosis, a binary logistic regression model was used. To compare overall survival between groups according to the presence of significant steatosis and advanced fibrosis, Kaplan-Meier survival curves were plotted, and log-rank test was utilized.

Graph Pad Prism Version 8 (San Diego, California, USA) was used for plotting Kaplan-Meier survival curves.

SPSS Version 24 (IBM, New York, USA) was used for all other statistical analyses. A $p$ value $<0.15$ was considered as trend, and a $p$ value $<0.05$ denoted statistical significance.

\section{Results}

\section{Differences in Baseline Characteristics Between Patients With and Without Significant Liver Steatosis (Table 1)}

Lean patients with significant steatosis (defined as $\geq 268 \mathrm{~dB} / \mathrm{m}$ in CAP; steatosis grades $\mathrm{S} 2 / \mathrm{S} 3$ ) were compared to patients without steatosis $(<268 \mathrm{~dB} / \mathrm{m} ; \mathrm{S} 0 / \mathrm{S} 1)$. Patients with steatosis had had higher GGT $(238.0( \pm 450.3)$ in S2/S3 steatosis vs. $112.1( \pm 180.0) \mathrm{U} / \mathrm{L}$ in S0/S1 steatosis; $p=0.013)$ and transaminase levels (ALT $59.1( \pm 58.8)$ vs. $44.3( \pm 52.7) \mathrm{U} / \mathrm{L}, p=0.048$; AST 63 ( \pm 67.4$)$ vs. 38.5 $( \pm 32.9) \mathrm{U} / \mathrm{L}, p=0.001)$. Regarding the metabolic profile, patients with steatosis had significantly higher triglyceride levels $(120.1( \pm 80.3) \mathrm{mg} / \mathrm{dL}$ in $\mathrm{S} 2 / \mathrm{S} 3$ steatosis vs. 96.1 $( \pm 58.2) \mathrm{mg} / \mathrm{dL}$ in $\mathrm{S} 0 / \mathrm{S} 1$ steatosis; $p=0.014)$ and higher serum uric acid levels $(5.3( \pm 1.5) \mathrm{mg} / \mathrm{dL}$ in $\mathrm{S} 2 / \mathrm{S} 3$ steatosis vs. $4.6( \pm 1.4) \mathrm{mg} / \mathrm{dL}$ in $\mathrm{S} 0 / \mathrm{S} 1$ steatosis; $p=0.047)$.

There were no differences in age, length of follow-up, total cholesterol, serum sodium, platelets, serum bilirubin, serum albumin, distribution of sex, presence of arterial hypertension, or kidney function.

\section{Risk Factors for Significant Steatosis (Table 2)}

In order to investigate independent risk factors for steatosis, we calculated uni- and multivariate binary logistic regression analyses. In univariate analysis, liver stiffness, presence of arterial hypertension, GGT, transaminases 
Table 1 Baseline characteristics in patients with and without significant steatosis
Table 2 Binary logistic regression analysis on risk factors for significant steatosis in lean patients $(\mathrm{CAP} \geq 268 \mathrm{~dB} / \mathrm{m})$

\begin{tabular}{lccc}
\hline & CAP $<268 \mathrm{~dB} / \mathrm{m}(n=184)$ & $\mathrm{CAP} \geq 268 \mathrm{~dB} / \mathrm{m}(n=97)$ & $p$ value \\
\hline Age (Years) & $45.3( \pm 15.7)$ & $47.8( \pm 15.6)$ & 0.200 \\
Female sex (\% female) & $103(56.0 \%)$ & $48(49.5 \%)$ & 0.316 \\
Arterial hypertension & $23(12.5 \%)$ & $19(19.6 \%)$ & 0.117 \\
Patients on diuretics $(\%)$ & $24(13.0 \%)$ & $14(14.4 \%)$ & 0.746 \\
Patients on allopurinol $(\%)$ & $0(0 \%)$ & $0(0 \%)$ & - \\
Serum uric acid $(\mathrm{mg} / \mathrm{dL})$ & $4.6( \pm 1.4)$ & $5.3( \pm 1.5)$ & $\mathbf{0 . 0 4 7}$ \\
Hyperuricemia ${ }^{\mathrm{a}}(\mathrm{n}, \%)$ & $8(4.3 \%)$ & $6(6.2 \%)$ & 0.568 \\
Stiffness $(\mathrm{kPa})$ & $12.0( \pm 15.7)$ & $15.6( \pm 19.5)$ & 0.115 \\
Sodium $(\mathrm{mmol} / \mathrm{L})$ & $140.0( \pm 2.6)$ & $140.2( \pm 4.1)$ & 0.733 \\
eGFR (mL/min) & $98.8( \pm 25.2)$ & $1.0( \pm 1.1)$ & 0.066 \\
Serum bilirubin $(\mathrm{mg} / \mathrm{dL})$ & $0.8( \pm 1.0)$ & $42.5( \pm 5.7)$ & 0.182 \\
Albumin $(\mathrm{mg} / \mathrm{dL})$ & $43.3( \pm 4.6)$ & $238.0( \pm 450.3)$ & 0.286 \\
Gamma-GT $(\mathrm{U} / \mathrm{L})$ & $112.1( \pm 180.0)$ & $59.1( \pm 58.8)$ & $\mathbf{0 . 0 1 3}$ \\
ALT (U/L) & $44.3( \pm 52.7)$ & $63( \pm 67.4)$ & $\mathbf{0 . 0 4 8}$ \\
AST (U/L) & $38.5( \pm 32.9)$ & $222.6( \pm 102.9)$ & $\mathbf{0 . 0 0 1}$ \\
Platelet count $(\mathrm{G} / \mathrm{L})$ & $223.6( \pm 94.8)$ & $187.1( \pm 51.8)$ & 0.939 \\
Total cholesterol $(\mathrm{mg} / \mathrm{dL})$ & $180( \pm 43.2)$ & $120.1( \pm 80.3)$ & 0.313 \\
Triglycerides $(\mathrm{mg} / \mathrm{dL})$ & $96.1( \pm 58.2)$ & $39.9( \pm 10.3)$ & $\mathbf{0 . 0 1 4}$ \\
Follow-up (months) & $38.3( \pm 10.7)$ & & 0.227 \\
\hline
\end{tabular}

Significant $p$ values are given in bold $(p<0.05)$

Continuous variables are presented as mean $( \pm \mathrm{SD})$

$C A P$ controlled attenuation parameters, $e G F R$ estimated glomerular filtration rate, $A L T$ alanine aminotransferase, $A S T$ aspartate aminotransferase

${ }^{a}$ Hyperuricemia was defined as $>7 \mathrm{mg} / \mathrm{dL}$ in men and $>5.7 \mathrm{mg} / \mathrm{dL}$ in women

\begin{tabular}{|c|c|c|c|c|c|c|c|c|}
\hline \multirow[t]{3}{*}{ Parameter } & \multicolumn{4}{|c|}{ Univariate analysis } & \multicolumn{4}{|c|}{ Multivariate analysis } \\
\hline & \multirow[t]{2}{*}{ OR } & \multicolumn{2}{|c|}{$95 \% \mathrm{CI}$} & \multirow[t]{2}{*}{$P$ value } & \multirow[t]{2}{*}{ OR } & \multicolumn{2}{|c|}{$95 \% \mathrm{CI}$} & \multirow[t]{2}{*}{$P$ value } \\
\hline & & Lower & Upper & & & Lower & Upper & \\
\hline Age (years) & 1.010 & 0.995 & 1.027 & 0.200 & & & & \\
\hline Sex (male vs. female) & 1.298 & 0.793 & 2.125 & 0.300 & & & & \\
\hline Liver stiffness (kPa) & 1.012 & 0.998 & 1.026 & 0.095 & 1.006 & 0.976 & 1.037 & 0.702 \\
\hline Arterial hypertension & 1.705 & 9.877 & 3.316 & 0.116 & 0.734 & 0.100 & 5.392 & 0.761 \\
\hline Gamma-GT (per U/L) & 1.002 & 1.000 & 1.003 & 0.008 & 1.003 & 0.999 & 1.006 & 0.141 \\
\hline ALT (per U/L) & 1.005 & 1.000 & 1.009 & 0.051 & 1.006 & 0.984 & 1.029 & 0.595 \\
\hline Serum cholesterol (per mg/dL) & 1.003 & 0.997 & 1.009 & 0.284 & & & & \\
\hline Triglycerides (per mg/dL) & 1.005 & 1.001 & 1.009 & 0.012 & 1.004 & 0.993 & 1.015 & 0.505 \\
\hline Serum uric acid (per mg/dL) & 1.288 & 0.945 & 1.755 & 0.109 & 1.434 & 1.001 & 2.054 & 0.049 \\
\hline
\end{tabular}

Significant $p$ value is given in bold $(p<0.05)$

$C A P$ controlled attenuation parameters, $A L T$ alanine aminotransferase
(ALT and AST), triglycerides, and serum uric acid showed a trend $(p<0.15)$ toward a significant influence on the risk of steatosis. In multivariate analysis, however, serum uric acid was the only independent risk factor for the presence of steatosis (OR 1.434, 95\% CI 1.001-2.054; $p=0.049$ ).

\section{Impact of Advanced Fibrosis and Steatosis on Overall Survival (Fig. 2)}

To identify risk factors for overall survival, we compared patients with advanced fibrosis or significant steatosis only, 
Fig. 2 Kaplan-Meier survival curve. Groups were compared after stratifying for the presence or absence of advanced liver fibrosis and hepatic steatosis. Advanced fibrosis was defined as $\mathrm{LSM} \geq 15 \mathrm{kPa}$, while hepatic steatosis was defined as $\mathrm{CAP} \geq 268 \mathrm{~dB} / \mathrm{m}$. Abbreviations: LSM, liver stiffness measurement; CAP, controlled attenuation parameters

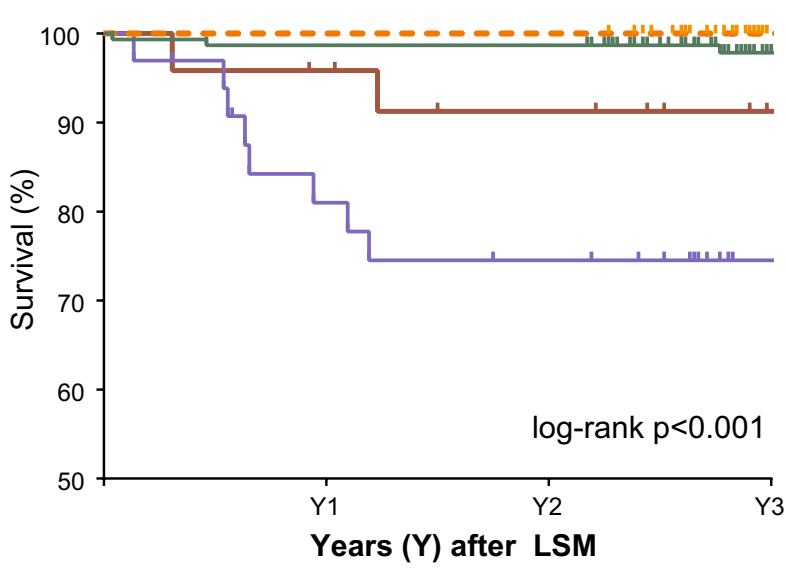

\# of patients at risk no fibrosis, no steatosis 151 steatosis only fibrosis and steatosis fibrosis only

151
73
24
33

$\perp$ no fibrosis, no steatosis

\lrcorner steatosis only

- fibrosis and steatosis

$\perp$ fibrosis only

as well as patients with steatosis and advanced fibrosis. During a mean follow-up of 38.9 ( \pm 10.6$)$ months, $14 / 281(5.0 \%)$ patients died. Patients with advanced fibrosis but no steatosis had the worst survival after 1, 2, and 3 years, while patients without significant steatosis or fibrosis or steatosis only had the highest survival rate $(p<0.001)$. Survival after 1,2 , and 3 years after LSM and CAP measurement was $98.7 \%$, $98.7 \%$, and $97.8 \%$ in patients without advanced fibrosis or significant steatosis, $98.6 \%, 98.6 \%$, and $98.6 \%$ in patients with significant steatosis only, $95.8 \%, 91.7 \%$, and $91.7 \%$ in patients with advanced fibrosis and significant steatosis, and $84.8 \%, 78.8 \%$, and $74.6 \%$ in patients with advanced fibrosis only (Fig. 2). Baseline characteristics of the subgroups of patients with advanced fibrosis ( $\geq 15 \mathrm{kPa}$ ) are presented in supplementary Table $\mathrm{S} 1$ and were not significantly different between patients with or without concomitant steatosis. In summary, advanced fibrosis rather than steatosis impacted overall survival in lean patients.

\section{Discussion}

The clinical impact of steatosis on survival in lean patients with or without underlying liver diseases remains unclear: While several studies have suggested that NAFLD is associated with impaired glucose tolerance and importantly, increased cardiovascular risk, data on the impact of simple steatosis in patients with physiological BMI values (i.e., lean subjects) are scarce. Many available population-based studies used conventional ultrasound (rather than CAP-based assessment) to identify significant steatosis in patients and did not provide information on fibrosis severity, which in fact, has been previously associated with cardiovascular risk and mortality in many patient cohorts [4, 9, 18].
A South Korean study published by Sinn et al. including 51,463 adults without diabetes mellitus or known liver disease found an increased risk for developing diabetes mellitus in lean patients with NAFLD (hazard ratio 1.18) during a median follow-up of 4 years [13]. In line with these findings, another large cohort study conducted in South Korea by Sung et al. found a significantly increased risk for developing diabetes mellitus in non-centrally obese patients with a BMI $\leq 25 \mathrm{~kg} / \mathrm{m}^{2}$ who had NAFLD [14]. However, in the referenced studies no data on overall mortality or the impact of hepatic steatosis (in lean patients) on liver-related death are available, and both studies only used noninvasive scores to estimate fibrosis severity, such as the NAFLD fibrosis score (NFS) and the FIB-4 score. Both scores are useful to screen for significant fibrosis but do not allow for an exact grading of fibrosis severity to the same extent as transient elastography or liver biopsy. Thus, we used liver stiffness measurement for an accurate identification of patients with advanced fibrosis. In addition, in our study the majority of patients were Caucasian, representing a different ethnicity and possibly different risk factors for lean NAFLD [19].

While it is commonly accepted that NAFLD is associated with impaired glucose tolerance/diabetes mellitus and mostly occurring in obese patients, several recently published studies tried to investigate the underlying causes for NAFLD/steatosis in lean patients [20]. Duarte et al. showed in a small cohort of NASH patients that there were significant differences in gut microbiome composition between lean, overweight, and obese NASH patients [20]. In a large study, Yun et al. investigated the differences in blood and gut microbiota between lean and obese NAFLD patients and also found considerable differences in microbiome composition [21]. These data further suggest that the gut-liver axis is of major pathophysiological importance in NAFLD 
and NASH. Similarly, a recent multicenter study addressed the interplay between bile acids, fibrosis, and gut microbiota composition and demonstrated that bile acid levels, which can alter the microbiome, were significantly higher in patients with NAFLD and additional fibrosis than in patients without fibrosis [22]. These data underline that fibrosis severity has to be considered in NAFLD/NASH studies as a major confounding factor. Unfortunately, no data on serum uric acid levels were presented in the referenced studies. However, recent evidence suggests that serum uric acid levels and purine metabolism are associated with NAFLD development [23]. Consequently, further studies are required to dissect this complex relationship [24]. In our study, next to uric acid, only triglycerides and transaminase levels were significantly different between lean patients with or without significant steatosis.

As outlined above, it remains unclear whether "isolated" steatosis impacts on survival [10]. Additionally, there are controversial results regarding the question if "lean NAFLD" has a worse survival than "obese NAFLD." The first report suggesting a different survival of lean versus obese NAFLD patients has yet not been published as a full manuscript [15]. Since then, more evidence has become available: A recent US study by Golabi et al. utilized data derived from the population-based NHANES III study and showed that lean NAFLD was associated with increased mortality, as compared to a lean control group [11]. During a followup of 229 months in the overall cohort, all-cause mortality significantly differed between the groups and was reported as $40.9 \%$ in lean NAFLD patients and $17.9 \%$ in lean control patients $(p<0.001)$. After adjusting for several variables, lean NAFLD remained significantly associated with allcause mortality (adjusted hazard ratio 1.54) [11].

In a Swedish study by Hagström et al. [12], biopsy-proven NAFLD patients were grouped according to their BMI. Ultimately, lean patients were older, had higher levels of transaminases, lower stages of fibrosis as well as a lower prevalence of NASH than the overweight/obese NAFLD patients. When analyzing overall mortality, no increased risk was observed in lean NAFLD patients as compared to ageand sex-matched obese NAFLD patients (hazard ratio 1.06; $p=0.73$ ) [12]. Of note, however, progression from mild to severe fibrosis seemed to be accelerated in lean NAFLD patients as compared to NAFLD patients with elevated BMI $[12,25]$. These previous findings are of relevance, as our study demonstrates that fibrosis rather than steatosis is the main driver of mortality in lean patients. As our patient cohorts are well characterized and survival data were derived from the national death registry, there is little risk of bias, strongly suggesting that fibrosis severity must always be taken into account when investigating (overall) survival in patients with steatosis. Importantly, our study has limitations. First, the gold standard for the evaluation of steatosis is liver biopsy, which was not generally available in our study. However, CAP-based steatosis assessment has a high accuracy, especially in lean patients [26]. Second, although manifest diabetes could be ruled out in all patients, impaired glucose tolerance could not be assessed in our cohort due to the retrospective design of our study. Finally, the follow-up period was rather short in order to allow definitive conclusions on cardiovascular morbidity/mortality or liver-related mortality that may occur due to an accelerated development of advanced fibrosis later on.

In conclusion, we could demonstrate that serum uric acid levels are independently associated with the presence of significant steatosis in lean patients, and that in these patients advanced fibrosis rather than steatosis itself leads to impaired overall survival.

Acknowledgments Open access funding provided by Medical University of Vienna.

Author's contribution LWU and TR designed the study; LWU, BF, MoMu, KS, EE, BS, NP, and RP performed data acquisition; LWU, $\mathrm{MaMa}$, and TR performed data and statistical analysis and interpretation; LWU and TR drafted the manuscript; all authors critically revised the manuscript for important intellectual content; MT and EE provided administrative and technical support. TR supervised the study.

Funding This research received no specific funding.

\section{Compliance with Ethical Standards}

Conflict of interest Lukas W. Unger, Bernadette Forstner, Moritz Muckenhuber, Katharina Scheuba, Ernst Eigenbauer, Bernhard Scheiner, Nikolaus Pfisterer, and Rafael Paternostro have no conflicts of interest to declare. Michael Trauner has served a speaker for BMS, Falk Foundation, Gilead and MSD; advisory boards for Albireo, Falk Pharma GmbH, Genfit, Gilead, Intercept, MSD, Novartis, Phenex and Regulus. He further received travel grants from Abbvie, Falk, Gilead and Intercept and unrestricted research grants from Albireo, Cymabay, Falk, Gilead, Intercept, MSD and Takeda. He is also co-inventor of patents on the medical use of norUDCA filed by the Medical University of Graz. Mattias Mandorfer has served as a speaker and/or consultant and/or advisory board member for AbbVie, Bristol-Myers Squibb, Gilead, and W. L. Gore \& Associates. Thomas Reiberger has served as a speaker and/or consultant and/or advisory board member for $\mathrm{Ab}-$ bVie, Bayer, Boehringer Ingelheim, Gilead, W. L. Gore \& Associates, and MSD and has received research funding from AbbVie, Boehringer Ingelheim, Gilead, MSD, Phenex Pharmaceuticals, and Philips.

Open Access This article is licensed under a Creative Commons Attribution-NonCommercial 4.0 International License, which permits any non-commercial use, sharing, adaptation, distribution and reproduction in any medium or format, as long as you give appropriate credit to the original author(s) and the source, provide a link to the Creative Commons licence, and indicate if changes were made. The images or other third party material in this article are included in the article's Creative Commons licence, unless indicated otherwise in a credit line to the material. If material is not included in the article's Creative Commons licence and your intended use is not permitted by statutory regulation or exceeds the permitted use, you will need to obtain permission 
directly from the copyright holder. To view a copy of this licence, visit http://creativecommons.org/licenses/by-nc/4.0/.

\section{References}

1. Collaborators GBD. Health effects of overweight and obesity in 195 countries over 25 Years. N Engl J Med. 2017;377:13-27.

2. Williams CD, Stengel J, Asike MI, et al. Prevalence of nonalcoholic fatty liver disease and nonalcoholic steatohepatitis among a largely middle-aged population utilizing ultrasound and liver biopsy: a prospective study. Gastroenterology. 2011;140:124-131.

3. Younossi ZM, Blissett D, Blissett R, et al. The economic and clinical burden of nonalcoholic fatty liver disease in the United States and Europe. Hepatology. 2016;64:1577-1586.

4. Unger LW, Forstner B, Schneglberger S, et al. Guideline-conform statin use reduces overall mortality in patients with compensated liver disease. Sci Rep. 2019;9:11674.

5. Zhang T, Zhang C, Zhang Y, et al. Metabolic syndrome and its components as predictors of nonalcoholic fatty liver disease in a northern urban Han Chinese population: a prospective cohort study. Atherosclerosis. 2015;240:144-148.

6. Chen $\mathrm{CH}$, Huang $\mathrm{MH}$, Yang JC, et al. Prevalence and risk factors of nonalcoholic fatty liver disease in an adult population of taiwan: metabolic significance of nonalcoholic fatty liver disease in nonobese adults. J Clin Gastroenterol. 2006;40:745-752.

7. Pu K, Wang Y, Bai S, et al. Diagnostic accuracy of controlled attenuation parameter (CAP) as a non-invasive test for steatosis in suspected Nonalcoholic fatty liver disease: a systematic review and meta-analysis. BMC Gastroenterol. 2019;19:51.

8. Hagstrom H, Elfwen O, Hultcrantz R, Stal P. Steatohepatitis is not associated with an increased risk for fibrosis progression in nonalcoholic fatty liver disease. Gastroenterol Res Pract. 2018;2018:1942648.

9. Angulo P, Kleiner DE, Dam-Larsen S, et al. Liver fibrosis, but no other histologic features, is associated with long-term outcomes of patients with nonalcoholic fatty liver disease. Gastroenterology. 2015;149(389-397):e310.

10. Scheiner B, Steininger L, Semmler G et al. Controlled attenuation parameter does not predict hepatic decompensation in patients with advanced chronic liver disease. Liver Int. 2019;39(1):127135. https://doi.org/10.1111/liv.13943.

11. Golabi P, Paik J, Fukui N, Locklear CT, de Avilla L, Younossi ZM. Patients with lean nonalcoholic fatty liver disease are metabolically abnormal and have a higher risk for mortality. Clin Diabetes. 2019;37:65-72.

12. Hagstrom H, Nasr P, Ekstedt M, et al. Risk for development of severe liver disease in lean patients with nonalcoholic fatty liver disease: a long-term follow-up study. Hepatol Commun. 2018;2:48-57.

13. Sinn DH, Kang D, Cho SJ et al. Lean nonalcoholic fatty liver disease and development of diabetes: a cohort study. Eur J Endocrinol. 2019. https://doi.org/10.1530/EJE-19-0143.
14. Sung KC, Seo DC, Lee SJ, Lee MY, Wild SH, Byrne CD. Non alcoholic fatty liver disease and risk of incident diabetes in subjects who are not obese. Nutr Metab Cardiovasc Dis. 2019;29:489-495.

15. Wattacheril J, Sanyal AJ. Lean NAFLD: an underrecognized outlier. Curr Hepatol Rep. 2016;15:134-139.

16. Reiberger T, Ferlitsch A, Payer BA, et al. Noninvasive screening for liver fibrosis and portal hypertension by transient elastography - a large single center experience. Wien Klin Wochenschr. 2012;124:395-402.

17. de Franchis R, Baveno VIF. Expanding consensus in portal hypertension: Report of the Baveno VI Consensus Workshop: Stratifying risk and individualizing care for portal hypertension. $J$ Hepatol. 2015;63:743-752.

18. Mangla N, Ajmera VH, Caussy $\mathrm{C}$ et al. Liver stiffness severity is associated with increased cardiovascular risk in patients with type 2 diabetes. Clin Gastroenterol Hepatol. 2019.

19. Younossi Z, Anstee QM, Marietti M, et al. Global burden of NAFLD and NASH: trends, predictions, risk factors and prevention. Nat Rev Gastroenterol Hepatol. 2018;15:11-20.

20. Duarte SMB, Stefano JT, Miele L, et al. Gut microbiome composition in lean patients with NASH is associated with liver damage independent of caloric intake: a prospective pilot study. Nutr Metab Cardiovasc Dis. 2018;28:369-384.

21. Yun Y, Kim HN, Lee EJ, et al. Fecal and blood microbiota profiles and presence of nonalcoholic fatty liver disease in obese versus lean subjects. PLoS One. 2019;14:e0213692.

22. Chen F, Esmaili S, Rogers G et al. Lean NAFLD: a distinct entity shaped by differential metabolic adaptation. Hepatology. 2019. https://doi.org/10.1002/hep.30908.

23. Gong S, Song J, Wang L, Zhang S, Wang Y. Hyperuricemia and risk of nonalcoholic fatty liver disease: a systematic review and meta-analysis. Eur J Gastroenterol Hepatol. 2016;28:132-138.

24. Jaruvongvanich V, Ahuja W, Wirunsawanya K, Wijarnpreecha K, Ungprasert P. Hyperuricemia is associated with nonalcoholic fatty liver disease activity score in patients with nonalcoholic fatty liver disease: a systematic review and meta-analysis. Eur J Gastroenterol Hepatol. 2017;29:1031-1035.

25. VanWagner LB, Armstrong MJ. Lean NAFLD: A not so benign condition? Hepatol Commun. 2018;2:5-8.

26. Vuppalanchi R, Siddiqui MS, Van Natta ML, et al. Performance characteristics of vibration-controlled transient elastography for evaluation of nonalcoholic fatty liver disease. Hepatology. 2018;67:134-144.

Publisher's Note Springer Nature remains neutral with regard to jurisdictional claims in published maps and institutional affiliations. 